\title{
BMJ
}

\section{Effect of a very low energy diet on moderate and severe obstructive sleep apnoea in obese men: a randomised controlled trial}

\author{
Kari Johansson, PhD student, ${ }^{1}$ Martin Neovius, postdoctoral research fellow, ${ }^{1,2}$ Ylva Trolle Lagerros, \\ physician, ${ }^{1,2}$ Richard Harlid, physician, ${ }^{3}$ Stephan Rössner, professor, ${ }^{1}$ Fredrik Granath, statistican, ${ }^{2}$ Erik \\ Hemmingsson, postdoctoral research fellow ${ }^{1}$
}

Obesity Unit, Department of Medicine, Karolinska Institutet Stockholm, Sweden

${ }^{2}$ Clinical Epidemiology Unit, Department of Medicine,

Karolinska Institutet

${ }^{3}$ Aleris Fysiologlab, Stockholm

Correspondence to: $\mathrm{K}$ Johansson, Obesity Unit, M73, Karolinska Institutet, Department of Medicine, SE-141 86 Stockholm, Sweden kari.johansson@ki.se

Cite this as: BMJ 2009;339:b4609 doi:10.1136/bmi.b4609

\section{ABSTRACT}

Objective To assess the effect of weight loss induced by a very low energy diet on moderate and severe obstructive sleep apnoea in obese men.

Design Single centre, two arm, parallel, randomised, controlled, open label trial. Blocked randomisation procedure used for treatment allocation.

Setting Outpatient obesity clinic in a university hospital in Stockholm, Sweden.

Participants 63 obese men (body mass index 30-40, age 30-65 years) with moderate to severe obstructive sleep apnoea (apnoea-hypopnoea index $(\mathrm{AHI}) \geq 15$ ), treated with continuous positive airway pressure.

Interventions The intervention group received a liquid very low energy diet ( $2.3 \mathrm{MJ} /$ day) for seven weeks to promote weight loss, followed by two weeks of gradual introduction of normal food, reaching 6.3 MJ/day at week 9. The control group adhered to their usual diet during the nine weeks of follow-up.

Main outcome measure $\mathrm{AHI}$, the major disease severity index for obstructive sleep apnoea. Data from all randomised patients were included in an intention to treat analysis (baseline carried forward for missing data). Results Of the 63 eligible patients, 30 were randomised to intervention and 33 to control. Two patients in the contro group were dissatisfied with allocation and immediately discontinued. All other patients completed the trial. Both groups had a mean AHI of 37 events/h (SD 15) at baseline. At week 9, the intervention group's mean body weight was $20 \mathrm{~kg}$ ( $95 \%$ confidence interval 18 to 21 ) lower than that of the control group, while its mean AHI was 23 events/h (15 to 30 ) lower. In the intervention group, five of $30(17 \%)$ were disease free after the energy restricted $\operatorname{diet}(\mathrm{AHI}<5)$, with 15 of 30 (50\%) having mild disease (AHI 5-14.9), whereas the $\mathrm{AHI}$ of all patients in the control group except one remained at 15 or higher. In a subgroup analysis of the intervention group, baseline AHI significantly modified the effectiveness of treatment, with a greater improvement in $\mathrm{AHI}$ in patients with severe obstructive sleep apnoea ( $\mathrm{AHI}>30)$ at baseline compared with those with moderate (AHI 15-30) sleep apnoea (AHI
$-38 v-12, P<0.001)$, despite similar weight loss $(-19.2 v$ $-18.2 \mathrm{~kg}, \mathrm{P}=0.55)$.

Conclusion Treatment with a low energy diet improved obstructive sleep apnoea in obese men, with the greatest effect in patients with severe disease. Long term treatment studies are needed to validate weight loss as a primary treatment strategy for obstructive sleep apnoea. Trial registration Current Controlled Trials ISRCTN70090382.

\section{INTRODUCTION}

Obstructive sleep apnoea is common and underdiagnosed, ${ }^{1}$ with estimated prevalences of $24 \%$ in middle aged men and $9 \%$ in middle aged women. ${ }^{23}$ Since obesity, old age, heredity, and male sex are known risk factors for the disorder, prevalence is expected to rise in the wake of the obesity epidemic. ${ }^{1}$ Left untreated, obstructive sleep apnoea confers an increased risk of driving related accidents, cardiovascular disease, sudden death during sleep, and all cause mortality. ${ }^{124-6}$

Few treatment options are available for obstructive sleep apnoea. The most commonly used strategy to reduce morbidity and mortality, and to improve quality of life, is continuous positive airway pressure to facilitate breathing during sleep. ${ }^{7}$ Although weight loss has been advocated as a primary treatment strategy for the condition, ${ }^{489}$ little high quality evidence exists to support this concept. Randomised controlled trials are especially lacking, prompting questions about selection bias and residual confounding in available studies. ${ }^{10}$ To the best of our knowledge, only one randomised controlled trial has been published on the effects of weight loss on obstructive sleep apnoea. ${ }^{11}$ However, the study did not include patients with moderate and severe disease, which, unlike the mild form, are both associated with increased mortality. ${ }^{56}$ Randomised studies are therefore needed to assess whether weight loss can improve moderate and severe obstructive sleep apnoea.

Our aim was, therefore, to assess potential improvement in obstructive sleep apnoea (defined using the 
apnoea-hypopnea index, AHI; the average rate of apnoeas and hypopnoeas per hour of sleep), after weight loss resulting from a very low energy diet, in obese men with moderate or severe obstructive sleep apnoea.

\section{METHODS}

\section{Participants}

The study was conducted at the Obesity Unit at Karolinska University Hospital, Karolinska Institute, Stockholm, Sweden, between February and April 2009.

Eligible for inclusion were men with moderate to severe obstructive sleep apnoea $(\mathrm{AHI} \geq 15)$ treated with continuous positive airway pressure for a minimum of six months, aged 30-65 years, body mass index (BMI) 30-40. We excluded those with contraindications for very low energy diets (1.9-3.4 MJ/day) according to the SCOOP-VLED report, ${ }^{12}$ type 1 or type 2 diabetes, current use of a weight loss drug, previous bariatric surgery, or recent angina pectoris or atrial fibrillation.

All patients were recruited from a patient database at the Aleris FysiologLab sleep clinic in Stockholm, Sweden. The database contained date of birth, height, weight, and data from nocturnal sleep studies in the past three years. A written invitation and screening questionnaire were sent to patients who met age, sex, $\mathrm{BMI}$, and AHI criteria (fig 1). Patients who declared an interest in participating and met the inclusion criteria were asked to attend a compulsory information meeting. Written informed consent was obtained from those still willing to participate at the end of the information meeting. Patients were screened by a physician to confirm absence of contraindications for a very low energy diet and to ensure that they were well informed about the trial aims.

\section{Intervention}

The intervention consisted of a weight loss programme starting with a seven week very low energy diet using a standard $2.3 \mathrm{MJ} /$ day liquid energy intake protocol (Cambridge Diet, Cambridge, Northants, UK), followed by two weeks of gradual introduction of normal food to reach $6.3 \mathrm{MJ}$ /day at week 9 . At weeks 1, 3, 5, 7, and 9 , basic clinical examinations were done, including weight, waist circumference, neck circumference, and percentage body fat. To confirm dietary compliance, urinary ketosis was assessed at each visit with test strips (Keto-Diabur-test 5000, Roche, Switzerland) for detection of acetoacetic acid (range 5-15 mmol/l). Each visit included a one hour group session to build group support and provide motivation. Group sessions were supervised by a research nurse and two study dietitians.

The patients in the control group were instructed to adhere to their usual diet. They also attended clinical examinations at weeks $1,3,5,7$, and 9 that were identical to those of the intervention group, except that group sessions were not provided. To reduce the likelihood of controls dropping out, they were offered the same weight loss programme as the intervention group once the nine week follow-up was completed. After the weight loss programme, all patients were offered a standard care one year weight maintenance programme.

\section{Primary outcome}

The primary outcome was AHI, the major disease severity index for obstructive sleep apnoea. AHI was assessed at baseline and after the intervention at week 9. Apnoea was defined as complete cessation of airflow for at least 10 seconds while hypopnoea was defined as a reduction in airflow for at least 10 seconds. ${ }^{13}$ The severity of obstructive sleep apnoea was defined by AHI, so that a frequency of five to 14.9 events per hour is considered mild, 15-30 events per hour moderate, more than 30 events per hour severe, and fewer than five events per hour, absent. ${ }^{13}$ In addition to AHI, oxygen desaturation episodes of $4 \%$ or more per hour of sleep, the nadir of arterial oxygen saturation, and percentage of supine time were recorded.

$\mathrm{AHI}$ and the other sleep measurements were derived from two consecutive nocturnal sleep studies in the home, using a six channel ambulatory polygraphy equipment (Watch PAT100, Itamar Medical Ltd, Caesarea, Israel). The equipment records peripheral arterial tone, heart rate, oxygen saturation, body position,

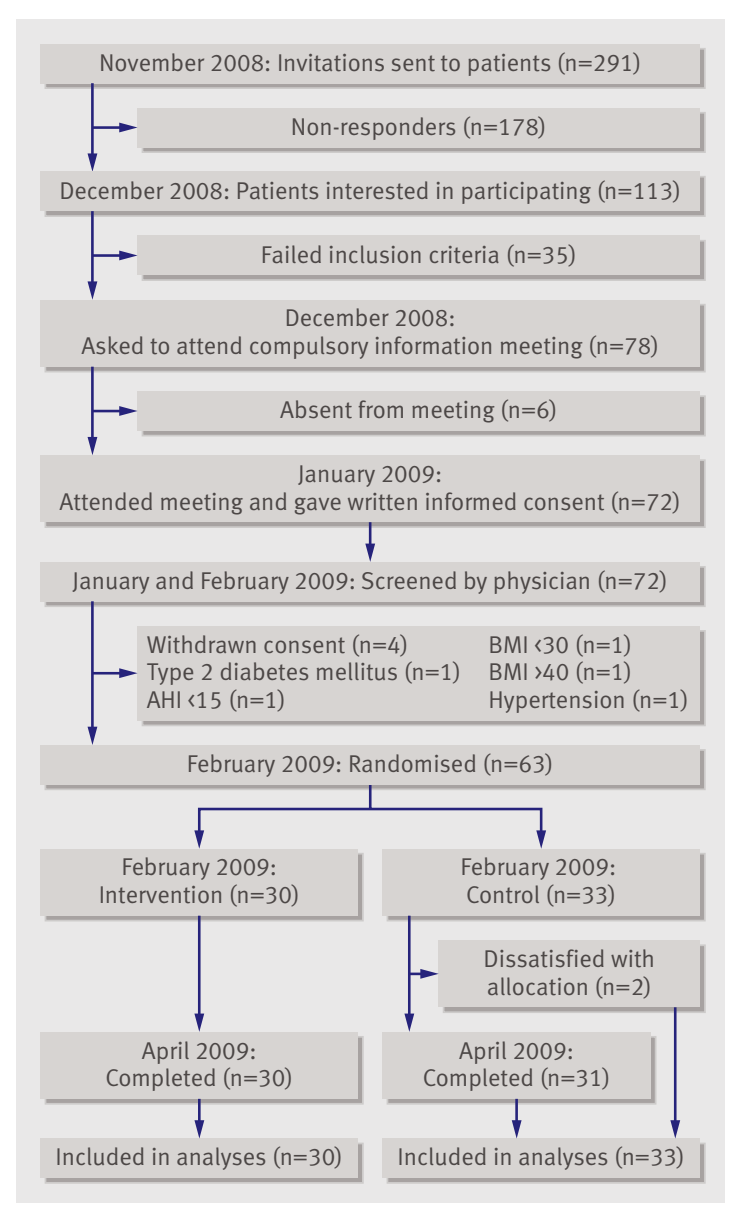

Fig 1 | Flow of participants. 
snoring, and actigraphy for automatic analysis of respiratory disturbance index, $\mathrm{AHI}$, and sleep-wake state. AHI measurement data were received automatically from the built-in software by a programmed mathematical algorithm. The algorithm was based on attenuation of the peripheral arterial tone in combination with heart rate and oxygen saturation excluding events during perceived wake periods. ${ }^{14}$ To decrease the effect of random variations, the average from the two consecutive nights was used for all the sleep measurements.

Patients were carefully instructed to refrain from using their continuous positive airway pressure device for the two nights before and during the nocturnal sleep studies. To avoid possible artefacts interfering with the automatic interpretation, such as arrhythmias, periodic limb movement, and related autonomic arousals, all sleep data were manually reviewed by a physician, who noted no major disturbances. Sleep studies with an estimated sleep time of less than three hours were excluded. At baseline, six patients did not record during one of the two nights (three intervention, three control), and seven patients did not record one of the two nights at follow-up (four intervention, three control).

\section{Secondary outcomes}

Fat loss was assessed with anthropometry and body composition changes from baseline at weeks 1, 3, 5, 7 , and 9 . Percentage body fat and body weight were measured with the Tanita BC-418MA body fat analyser (Tanita Corporation of America, Arlington Heights, IL, USA), which has been validated for measuring change in adiposity. ${ }^{15}$ Standing height was measured to the nearest centimetre with a wall mounted stadiometer. Waist circumference was measured in duplicate between the iliac crest and the lower rib cage, with patients standing in underwear. Neck circumference was measured in duplicate at the level of the superior border of the cricothyroid membrane.

Daytime sleepiness was assessed with the Epworth sleepiness scale, ${ }^{16}$ an eight item self administered questionnaire. The patients rated on a four point scale (0-3) the chances of dozing off or falling asleep in eight different situations. When responses are added they provide a daytime sleepiness score. A score of $0-9$ is considered normal, whereas a score of 10-24 indicates a possibility of excessive daytime sleepiness.

\section{Safety}

Adverse events from the very low energy diet were noted by the study nurse at each visit, and subsequently classified by the study physician for potential causality (unlikely, possibly, or likely). The most common adverse events from a very low energy diet are dry mouth, diarrhoea, constipation, headache, nausea or vomiting, dizziness, cramps, fatigue, hunger, visual disturbance, feeling cold, hair loss, gallstones, and gout, all of which are considered transient. ${ }^{12}$

\section{Randomisation}

Sixty three patients met the study inclusion criteria after the physician screening, and were subsequently randomly assigned to intervention or control groups in a 1:1 ratio. A member of the research team who was not involved in the recruitment process, was given a list containing only study identification number, AHI, and BMI. Each identification number was then put into one of four categories of AHI and BMI (low AHI and low BMI; low AHI and high BMI; high AHI and low $\mathrm{BMI}$; or high AHI and high BMI, using screening median values as cut offs). Each category contained a computer generated random sequence of 18 As (intervention) and $18 \mathrm{Bs}$ (control). Once the study identification numbers were in their correct category, they were sequentially allocated to intervention or control. The randomisation lists were kept in a locked room, with no access for recruitment staff. When all patients were randomised, a list of study identification numbers and allocation was sent to study administrators who informed the patients of their allocation. In view of the use of a liquid very low energy diet, blinding of staff or participants was not considered feasible.

\section{Sample size}

The sample size calculation was based on $80 \%$ power with an $\alpha$ level of $5 \%$ to detect a difference in AHI of 15 events an hour between groups using a two sided independent Student's $t$ test. We assumed a standard deviation of the within group AHI difference of 18 events per hour based on two previous studies. ${ }^{1718}$ This resulted in a required sample size of 24 patients in each group. To avoid loss of power due to dropout, we increased the final recruitment goal by $25 \%$, resulting in a recruitment target of 30 patients in each group.

\section{Statistical analysis}

Mean values and standard deviations were used to describe the baseline characteristics and within group changes in the two groups.

Changes were calculated by subtracting the baseline measurement from the nine week follow-up measurement. The mean difference in change between groups was used as main treatment effect along with $95 \%$ confidence intervals. Changes between groups from baseline to week 9 were analysed with independent samples $t$ test.

Effect modification by baseline AHI was investigated by fitting a linear regression model with $\mathrm{AHI}$ at week 9 as the outcome variable, and baseline AHI, treatment group (intervention or control), and an interaction term (baseline AHI $\times$ treatment group) as predictors.

Adiposity measures were also analysed with repeated measures ANOVA with group×time interaction.

All analyses were done on an intention to treat basis with baseline carried forward for missing data. A per protocol analysis was also done as a sensitivity analysis. Of 378 planned examinations in dietary compliance and adiposity measures, we were able to do 360 
(95\%) with all the missing data originating from the control group. All data were analysed with SPSS (version 17.0, Chicago, IL) and Stata (version 10, College Station, TX, USA).

\section{RESULTS}

Sixty three men were randomised to intervention $(n=30)$ or control $(n=33)$. All patients completed the trial apart from two control patients who were dissatisfied with allocation and immediately discontinued the trial (fig 1).

Table 1 shows the characteristics of the two groups. For both groups combined, mean age was 49 years (SD 7.3, range 33-61), mean AHI 37 events/h (15, 15-77), mean weight $112.5 \mathrm{~kg}(14.2,86.9-141.9)$, and mean waist circumference $120 \mathrm{~cm}(9,101-139)$. Forty four per cent of the patients had moderate obstructive sleep apnoea (AHI 15-30) and the remaining 56\% had severe obstructive sleep apnoea (AHI >30). Sixty per cent were class I obese (BMI 30.0-34.9) and 40\% were class II obese $(\mathrm{BMI} \geq 35)$.

\section{Compliance}

During the seven weeks of the very low energy diet, all patients in the intervention group complied with the diet, as assessed by urinary ketosis $(5-15 \mathrm{mmol} / \mathrm{l}$ at each visit). Ketosis was not present in any of the control patients during the nine weeks.

\section{Adiposity change}

At week 9, all four adiposity measures differed significantly between groups (figure 2 , all $\mathrm{P}<0.001$ ). In the intervention group, the mean change in weight was $-18.7 \mathrm{~kg}$ (SD 4.1; range -26.7 to -10.6 ) and mean change in BMI was $-5.7(1.1 ;-8.2$ to -3.4$)$, compared with $1.1 \mathrm{~kg}(1.9 ;-2$ to 6.2$)$ and $0.3(0.6 ;-0.6$ to 1.9$)$ in the control group. Twenty two of $30 \quad(73 \%)$

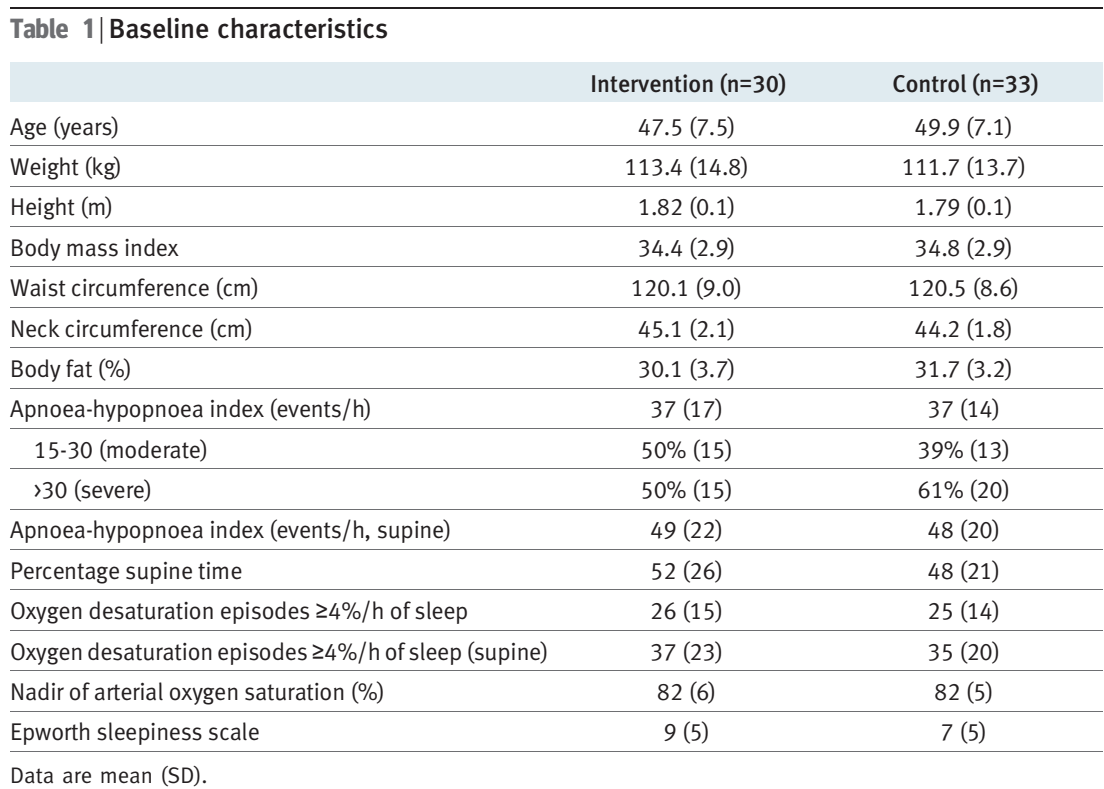

intervention patients were non-obese $(\mathrm{BMI}<30)$ at week 9 , whereas all control patients remained obese.

\section{Primary outcome}

At week 9, mean AHI in the intervention group was 12 (SD 7, range 1-30) compared with 35 (14, 14-75) in the control group (table 2). In the intervention group, a dose-response relation existed between weight loss
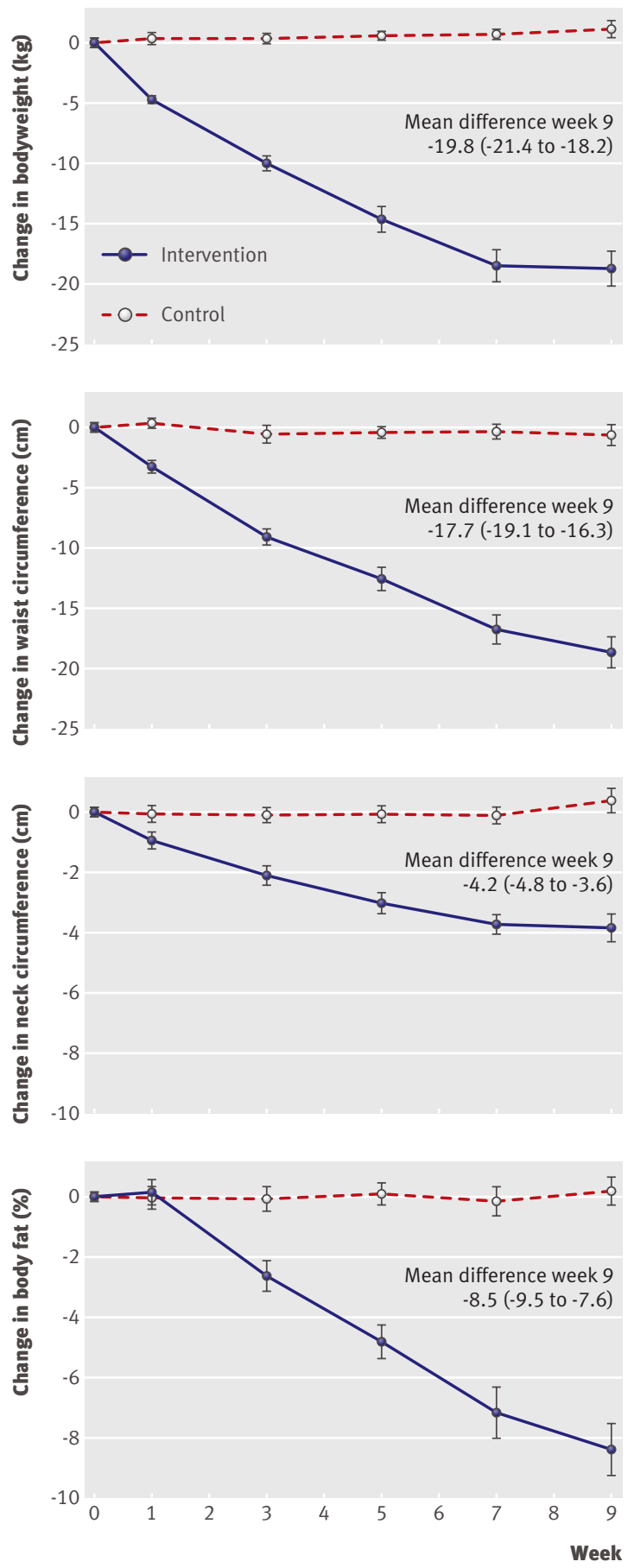

Fig 2 | Changes from baseline in body weight (A), waist circumference (B), neck circumference (C), and percentage body fat (D). Data are mean difference between groups $(95 \%$ confidence interval). P $<0.001$ for intervention $v$ control group for all comparisons. 
Table $2 \mid$ Changes in obstructive sleep apnoea measures between baseline and week 9

\begin{tabular}{|c|c|c|c|c|}
\hline & $\begin{array}{l}\text { Intervention } \\
\qquad(n=30)\end{array}$ & $\begin{array}{l}\text { Control } \\
(n=33)\end{array}$ & $\begin{array}{c}\text { Mean } \\
\text { difference }\end{array}$ & $\mathbf{P}$ \\
\hline $\mathrm{AHI}$ (events/h) & $-25(17)$ & $-2(11)$ & $-23(-30$ to -15$)$ & $<0.001$ \\
\hline AHI (events/h; supine) & $-27(25)$ & $-1(12)$ & $-27(-36$ to -17$)$ & $<0.001$ \\
\hline Percentage supine time & $-5(25)$ & $2(22)$ & $-7(-5$ to 18$)$ & 0.28 \\
\hline Oxygen desaturation episodes $\geq 4 \% / \mathrm{h}$ of sleep & $-19(14)$ & $-1(9)$ & $-18(-24$ to -12$)$ & $<0.001$ \\
\hline Oxygen desaturation episodes $\geq 4 \% / \mathrm{h}$ of sleep (supine) & $-23(23)$ & $-0(10)$ & $-23(-32$ to -14$)$ & $<0.001$ \\
\hline Nadir of arterial oxygen saturation (\%) & $5(6)$ & $0(5)$ & $5(2$ to 7$)$ & 0.002 \\
\hline Epworth sleepiness scale & $-3(5)$ & $1(3)$ & $-4(-6$ to -2$)$ & $<0.001$ \\
\hline
\end{tabular}

Data are mean (SD) for within group comparisons, and mean (95\% confidence interval) for between group comparisons. P values from independent samples $t$ tests. AHI=apnoea-hypopnoea index.

and change in $\mathrm{AHI}$, although its strength was moderate $(\mathrm{r}=0.4, \mathrm{P}=0.04$, appendix 1$)$.

Using AHI cut offs for mild, moderate, and severe obstructive sleep apnoea, 12 of $30(40 \%)$ in the intervention group improved by two categories of AHI, 14 of $30(47 \%)$ improved by one category, and four of 30 $(13 \%)$ remained in the same category. In the control group one of 33 (3\%) improved by two AHI categories, three of $33(9 \%)$ improved by one AHI category, 24 of 33 remained static (73\%), and five of 33 worsened to a higher AHI category (15\%) (fig 3).

Baseline AHI significantly modified the treatment effect $(\mathrm{P}<0.001$, figure 4 and appendix 2$)$ in the intervention group. In the intervention group, the change in $\mathrm{AHI}$ in patients with severe obstructive sleep apnoea at baseline was -38 and -12 for those with moderate disease $(\mathrm{P}<0.001$, control-group adjusted change of -33 for those with moderate disease and -14 for severe). This meant that the mean AHI at week 9 was similar for patients with moderate obstructive sleep apnoea at baseline as for those with severe disease at baseline (AHI $11 v$ 13, $\mathrm{P}=0.45$ ).

The modification of treatment effect by baseline AHI was not explained by differences between patients with moderate compared with severe sleep apnoea in any of the adiposity measurements at baseline, age, or weight loss achieved at week $9(-19.2 \mathrm{v}$ $-18.2 \mathrm{~kg}, \mathrm{P}=0.55)$ between. Furthermore, AHI at baseline did not correlate with any of the adiposity measures.

\section{Secondary sleep outcomes}

Apart from AHI, the intervention group also improved in other sleep variables compared with the control group (table 2). In terms of self reported outcomes, reductions in daytime sleepiness score at week 9 were greater in the intervention group than in the control group $(\mathrm{P}<0.001)$.

\section{Safety}

Eight adverse events were classified by the study physician as being likely to be causally linked with the very low energy diet. These were constipation $(n=3)$, elevated alanine aminotransferase concentrations $(n=2)$, dizziness $(\mathrm{n}=1)$, gout $(\mathrm{n}=1)$, and dry lips $(\mathrm{n}=1)$. All adverse events were transient and not present at week 9. No patient discontinued treatment due to adverse events and no adverse events occurred in the control group.

\section{Sensitivity analysis}

Use of a per protocol analysis, rather than intention to treat analysis with baseline carried forward, did not materially change our findings for any of the reported outcomes (appendix 3).
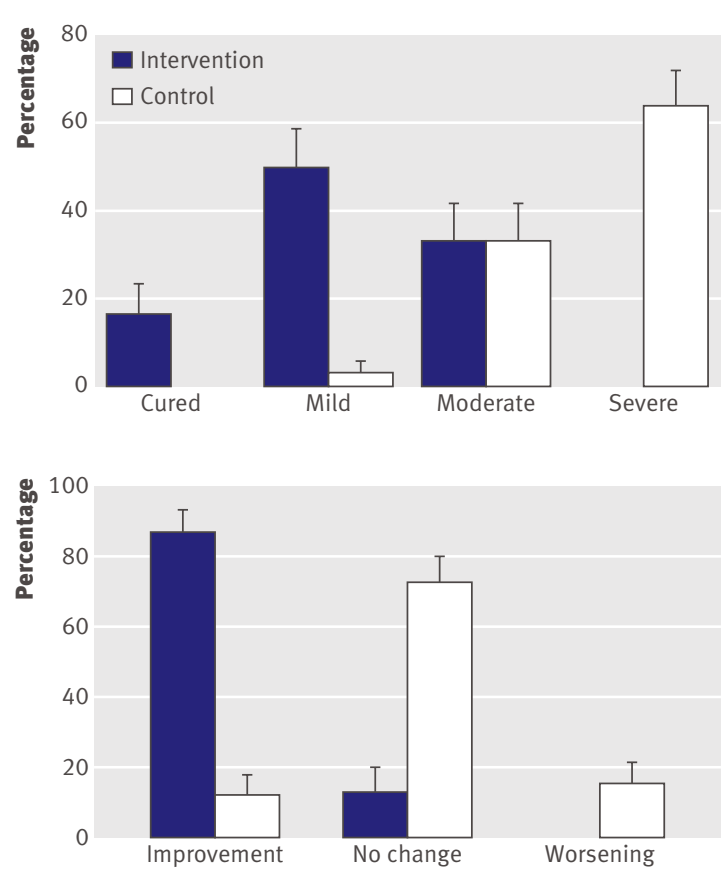

Fig $3 \mid$ Obstructive sleep apnoea at week 9. Proportions of patients defined as having no (cured), mild, moderate, or severe obstructive sleep apnoea at week 9 (A). Proportions of patients who improved, maintained, or worsened their obstructive sleep apnoea status after 9 weeks (B). Error bars are $95 \%$ confidence intervals. Definitions: Cured defined as AHI $<5$ events/h, mild 5-14.9 events/h, moderate AHI 15-30 events/h, severe $>30$ events/h. Improvement defined as shift in severity of obstructive sleep apnoea-that is, moderate to cured or mild; or severe to cured, moderate or mild.

Worsening defined as opposite direction of improvement. No change defined as remaining within the same category of severity of obstructive sleep apnoea. 


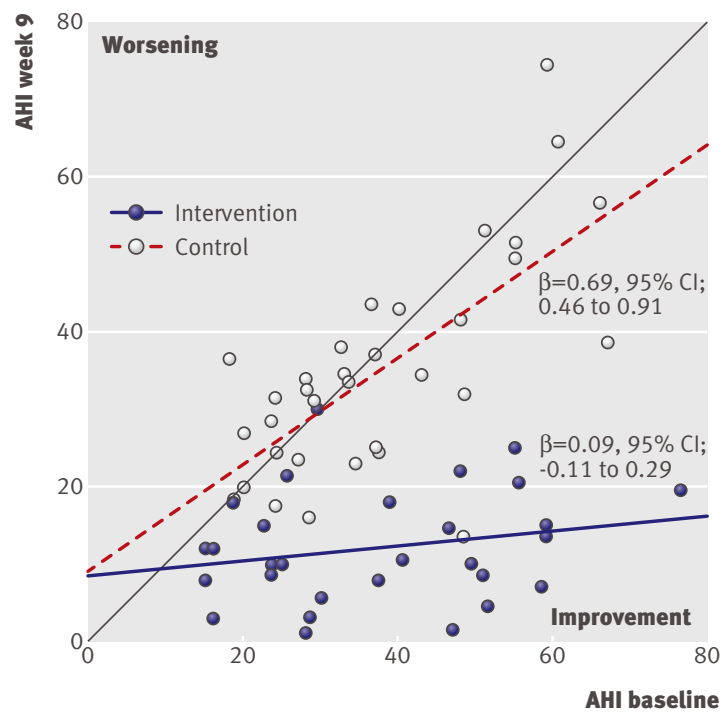

Fig 4 Association between AHI at baseline and follow-up for intervention group (thick blue line) and control group (broken red line). Thin black line represents no change in AHI from baseline

\section{DISCUSSION}

Treatment with a low energy diet resulted in a clinically meaningful reduction of moderate to severe obstructive sleep apnoea in obese men. AHI was reduced by $67 \%$ in the intervention group compared with no change in the control group. Of the patients in the intervention group, those with severe sleep apnoea at baseline had significantly greater reductions in AHI than did those starting with moderate sleep apnoea. Seventeen per cent of the intervention group patients were classified as free of obstructive sleep apnoea at week 9. Daytime sleepiness was also reduced in the intervention group. An added benefit of the treatment was that $73 \%$ of patients in the intervention group were no longer classified as obese, whereas all control patients remained obese.

\section{Strengths and weaknesses}

The strengths of this study include the randomised controlled design, which implies a low probability of selection bias and residual confounding. Furthermore, the study had a low dropout rate, with few missing data, ample statistical power, duplicated sleep studies, and investigation of a patient group with increased risk of mortality. Additionally, the low cost, low energy diet treatment programme used could potentially be implemented in primary care. The main limitation is the short study duration, which means that the long term effect of weight loss by a very low energy diet on moderate to severe obstructive sleep apnoea remains uncertain. Although the study supports a beneficial role of weight loss on obstructive sleep apnoea, the duration was not sufficient to constitute validation of treatment. Indeed, since weight regain is common after weight loss, ${ }^{19}$ weight loss trials purporting to be treatment studies of any obesity comorbidity should have a minimum duration of a year.
In terms of data quality, the gold standard method for diagnosing obstructive sleep apnoea is polysomnography. However, high costs, limited access to sleep laboratories, and the increasing number of patients with obstructive sleep apnoea, have led to the development of more accessible and cheaper methods, such as the portable monitors used in this study. Validation studies have shown that the manually portable devices based on peripheral arterial tone records have high sensitivity and specificity to identify pathological respiratory disturbances and estimate sleep time compared with polysomnography. ${ }^{1420}$

\section{Previous research}

Although obesity has been identified as a modifiable risk factor for obstructive sleep apnoea, ${ }^{1}$ few studies exist that investigate the effect of diet induced weight loss to treat the disease. ${ }^{10}$ Indeed, we could only identify one randomised intervention study that assessed the effect of weight loss on obstructive sleep apnoea. ${ }^{11}$ The authors in that study noted a $40 \%$ reduction in AHI from a weight loss of $10.7 \mathrm{~kg}$ after a year in patients with mild disease. Uncontrolled studies have also noted improvements in obstructive sleep apnoea (defined by oxygen desaturation episodes, $\geq 4 \%$ per hour of sleep) from weight loss following a very low energy diet, with weight loss ranging between 9-21 kg and improvements in oxygen desaturation ranging from 12 to 54 episodes per hour. ${ }^{21-25}$ However, these studies had small sample sizes (eight to 31 patients), and their designs were susceptible to bias and confounding (no control group and no randomisation). Despite these limitations, these studies indicate treatment effects of similar size to that seen in the current study and the previously published randomised controlled trial on mild obstructive sleep apnoea. ${ }^{11}$ Additionally, prospective cohort studies have shown that weight gain is associated with increased risk of sleep apnoea, and conversely that weight loss is associated with improvement in obstructive sleep apnoea. ${ }^{26-28}$

Future research priorities include intervention studies with longer duration so that the long term effectiveness of weight loss programmes can be clarified. Long term weight loss will also have a favourable effect on other obesity comorbidities, such as type 2 diabetes and cardiovascular disease, which often coexist with obstructive sleep apnoea. Indeed, a prevalence study showed that $87 \%$ of obese patients with type 2 diabetes had at least mild sleep apnoea. ${ }^{29}$

Another area that needs further study is whether amount of weight loss is associated with sleep apnoea improvement. In this trial, the association between weight loss and AHI improvement was significant, although of modest strength $(\mathrm{r}=0.4)$. This relation suggests that factors other than weight loss are also involved in the improvement of sleep apnoea.

\section{Clinical implications}

Arguments for large scale implementation of weight loss programmes by very low energy diet in the 


\section{WHAT IS ALREADY KNOWN ON THIS SUBJECT}

Unlike mild obstructive sleep apnoea, moderate and severe obstructive sleep apnoea are associated with increased all cause mortality

Weight loss has been advocated as a primary treatment option for obstructive sleep apnoea

Only one randomised controlled trial has been published, showing improvement in mild obstructive sleep apnoea after weight loss

\section{WHAT THIS STUDY ADDS}

Treatment with a low energy diet resulted in a significant reduction of moderate to severe obstructive sleep apnoea in obese men

Patients with severe obstructive sleep apnoea benefited most from the intervention.

treatment of obstructive sleep apnoea include the relative safety and effectiveness of a very low energy diet and the low cost compared with bariatric surgery. Bariatric surgery, currently the most effective method for treating obesity, results in weight loss of about $25-30 \mathrm{~kg}$ after 10 years. $^{30}$ Evidence is emerging that bariatric surgery is effective in treating obstructive sleep apnoea. $^{1831-33}$ However, since the prevalence of obstructive sleep apnoea is expected to rise in the wake of the obesity epidemic, ${ }^{1}$ provision of bariatric surgery to all patients with this problem is not realistic. Non-surgical treatment schemes, preferably of lower cost than bariatric surgery and with proved effectiveness, are therefore needed. The main argument against a weight loss programme using a very low energy diet is the uncertainty regarding weight loss maintenance. However, a systematic review of randomised weight loss trials with a minimum follow-up of a year showed that very low energy diets resulted in a greater $(10 \%)$ weight reduction from baseline weight after a year, compared with drug treatment $(8 \%) .{ }^{19}$ One promising approach to improve weight loss maintenance is to use anti-obesity drugs after the active weight loss phase. ${ }^{34-36}$ Whether such a treatment scheme would be effective in promoting long term improvements in obstructive sleep apnoea needs clarification.

In conclusion, the findings of this randomised trial show that weight loss induced by a very low energy diet improved moderate to severe obstructive sleep apnoea in obese men. Patients with severe sleep apnoea had greater improvements than those with moderate disease.

We thank the staff at the Obesity Unit, the staff at Aleris Fysiologlab for assistance with the sleep studies, and the participating patients. Contributors: KJ, MN, SR and EH conceived the study hypothesis. YLT and SR met all the patients. RH performed the sleep studies. KJ, MN, and FG did the statistical analyses. $\mathrm{KJ}$ and $\mathrm{EH}$ wrote the first draft of the manuscript. MN, SR, YLT, and RH provided critical input at all stages and critically reviewed and contributed to the final draft. All authors are guarantors

Funding: This study was partly supported by a grant from Cambridge Manufacturing Company Limited and Novo Nordisk Scandinavia AB. The funders played no part in the analysis or write up of this paper.

Competing interests: None declared.

Ethical approval: The regional ethics committee in Stockholm, Sweden, approved the study.

Data sharing: No additional data available.
1 Punjabi NM. The epidemiology of adult obstructive sleep apnea. Proc Am Thorac Soc 2008; 5:136-43.

2 Young T, Peppard PE, Gottlieb DJ. Epidemiology of obstructive sleep apnea: a population health perspective. Am J Respir Crit Care Med 2002;165:1217-39.

3 Young T, Palta M, Dempsey J, Skatrud J, Weber S, Badr S. The occurrence of sleep-disordered breathing among middle-aged adults. N Engl J Med 1993;328:1230-5.

4 Shaw IE, Punjabi NM, Wilding IP, Alberti KG, Zimmet PZ. Sleepdisordered breathing and type 2 diabetes: a report from the International Diabetes Federation Taskforce on Epidemiology and Prevention. Diabetes Res Clin Pract 2008;81:2-12.

5 Young T, Finn L, Peppard PE, Szklo-Coxe M, Austin D, Nieto FJ, et al. Sleep disordered breathing and mortality: eighteen-year follow-up of the Wisconsin sleep cohort. Sleep 2008;31:1071-8.

6 Marshall NS, Wong KK, Liu PY, Cullen SR, Knuiman MW, Grunstein RR. Sleep apnea as an independent risk factor for all-cause mortality: the Busselton Health Study. Sleep 2008;31:1079-85.

7 Banno K, Kryger MH. Sleep apnea: clinical investigations in humans. Sleep Med 2007;8:400-26.

8 de Sousa AG, Cercato C, Mancini MC, Halpern A. Obesity and obstructive sleep apnea-hypopnea syndrome. Obes Rev 2008;9:340-54.

9 Morgenthaler TI, Kapen S, Lee-Chiong T, Alessi C, Boehlecke B, Brown T, et al. Practice parameters for the medical therapy of obstructive sleep apnea. Sleep 2006;29:1031-5.

10 Veasey SC, Guilleminault C, Strohl KP, Sanders MH, Ballard RD, Magalang UJ. Medical therapy for obstructive sleep apnea: a review by the Medical Therapy for Obstructive Sleep Apnea Task Force of the Standards of Practice Committee of the American Academy of Sleep Medicine. Sleep 2006;29:1036-44.

11 Tuomilehto HP, Seppa JM, Partinen MM, Peltonen M, Gylling H, Tuomilehto JO, et al. Lifestyle intervention with weight reduction: first-line treatment in mild obstructive sleep apnea. Am J Respir Crit Care Med 2009;179:320-7.

12 SCOOP-VLCD Task 7.3. Scientific Co-operation on Questions Relating to Food: Directorate-General Health and Consumer Protection, European Union. http://ec.europa.eu/food/fs/scoop/7.3_en.pdf.

13 Sleep-related breathing disorders in adults: recommendations for syndrome definition and measurement techniques in clinical research. The Report of an American Academy of Sleep Medicine Task Force. Sleep 1999;22:667-89.

14 Bar A, Pillar G, Dvir I, Sheffy J, Schnall RP, Lavie P. Evaluation of a portable device based on peripheral arterial tone for unattended home sleep studies. Chest 2003;123:695-703.

15 Neovius M, Udden J, Hemmingsson E. Assessment of change in body fat percentage with DXA and eight-electrode BIA in centrally obese women. Med Sci Sports Exerc 2007;39:2199-203.

16 Johns MW. A new method for measuring daytime sleepiness: the Epworth sleepiness scale. Sleep 1991;14:540-5.

17 Kemppainen T, Ruoppi P, Seppa J, Sahlman J, Peltonen M, Tukiainen $\mathrm{H}$, et al. Effect of weight reduction on rhinometric measurements in overweight patients with obstructive sleep apnea. Am J Rhinol 2008;22:410-5.

18 Lettieri CJ, Eliasson AH, Greenburg DL. Persistence of obstructive sleep apnea after surgical weight loss. J Clin Sleep Med 2008;4:333-8.

19 Franz MJ, VanWormer JJ, Crain AL, Boucher JL, Histon T, Caplan W, et al. Weight-loss outcomes: a systematic review and meta-analysis of weight-loss clinical trials with a minimum 1-year follow-up. J Am Diet Assoc 2007;107:1755-67.

20 Zou D, Grote L, Peker Y, Lindblad U, Hedner J. Validation a portable monitoring device for sleep apnea diagnosis in a population based cohort using synchronized home polysomnography. Sleep 2006;29:367-74.

21 Hakala K, Maasilta P, Sovijarvi AR. Upright body position and weight loss improve respiratory mechanics and daytime oxygenation in obese patients with obstructive sleep apnoea. Clin Physiol 2000;20:50-5.

22 Kajaste S, Brander PE, Telakivi T, Partinen M, Mustajoki P. A cognitive-behavioral weight reduction program in the treatment of obstructive sleep apnea syndrome with or without initial nasal CPAP: a randomized study. Sleep Med 2004:5:125-31.

23 Kansanen M, Vanninen E, Tuunainen A, Pesonen P, Tuononen V, Hartikainen J, et al. The effect of a very low-calorie diet-induced weight loss on the severity of obstructive sleep apnoea and autonomic nervous function in obese patients with obstructive sleep apnoea syndrome. Clin Physiol 1998;18:377-85.

24 Lojander J, Mustajoki P, Ronka S, Mecklin P, Maasilta P. A nursemanaged weight reduction programme for obstructive sleep apnoea syndrome. J Intern Med 1998;244:251-5.

25 Suratt PM, McTier RF, Findley LI, Pohl SL, Wilhoit SC. Effect of verylow-calorie diets with weight loss on obstructive sleep apnea. Am J Clin Nutr 1992;56:182S-184S. 
26 Peppard PE, Young T, Palta M, Dempsey J, Skatrud J. Longitudinal study of moderate weight change and sleep-disordered breathing. JAMA 2000;284:3015-21.

27 Tishler PV, Larkin EK, Schluchter MD, Redline S. Incidence of sleepdisordered breathing in an urban adult population: the relative importance of risk factors in the development of sleep-disordered breathing. JAMA 2003;289:2230-7.

28 Newman AB, Foster G, Givelber R, Nieto F), Redline S, Young T. Progression and regression of sleep-disordered breathing with changes in weight: the Sleep Heart Health Study. Arch Intern Med 2005;165:2408-13.

29 Foster GD, Sanders MH, Millman R, Zammit G, Borradaile KE, Newman $A B$, et al. Obstructive sleep apnea among obese patients with type 2 diabetes. Diabetes Care 2009;32:1017-9.

30 Sjostrom L, Lindroos AK, Peltonen M, Torgerson J, Bouchard C, Carlsson B, et al. Lifestyle, diabetes, and cardiovascular risk factors 10 years after bariatric surgery. N Engl J Med 2004;351:2683-93.

31 Fritscher LG, Canani S, Mottin CC, Fritscher CC, Berleze D, Chapman K, et al. Bariatric surgery in the treatment of obstructive sleep apnea in morbidly obese patients. Respiration 2007;74:647-52.
32 Valencia-Flores M, Orea A, Herrera M, Santiago V, Rebollar V, Castano VA, et al. Effect of bariatric surgery on obstructive sleep apnea and hypopnea syndrome, electrocardiogram, and pulmonary arterial pressure. Obes Surg 2004;14:755-62.

33 Pillar G, Peled R, Lavie P. Recurrence of sleep apnea without concomitant weight increase 7.5 years after weight reduction surgery. Chest 1994;106:1702-4.

34 Apfelbaum M, Vague P, Ziegler O, Hanotin C, Thomas F, Leutenegger $\mathrm{E}$. Long-term maintenance of weight loss after a verylow-calorie diet: a randomized blinded trial of the efficacy and tolerability of sibutramine. Am J Med 1999;106:179-84.

35 Mathus-Vliegen EM. Long-term maintenance of weight loss with sibutramine in a GP setting following a specialist guided very-lowcalorie diet: a double-blind, placebo-controlled, parallel group study. Eur J Clin Nutr 2005;59(suppl 1):S31-8; discussion S39.

36 Richelsen B, Tonstad S, Rossner S, Toubro S, Niskanen L, Madsbad S, et al. Effect of orlistat on weight regain and cardiovascular risk factors following a very-low-energy diet in abdominally obese patients: a 3-year randomized, placebocontrolled study. Diabetes Care 2007;30:27-32

Accepted: 13 September 2009 